\title{
Assessment of bone resorption differences in patients rehabilitated with screw retained prosthesis and telescopic crowns: A pilot study
}

\author{
K. Sayed Akber Pasha ${ }^{1, *}$, Sandeep Bhatia ${ }^{2}$, Rajdeep Singh ${ }^{3}$ \\ ${ }^{1}$ Prosthodontist, ${ }^{2,3}$ Senior Lecturer, ${ }^{2}$ Dept. of Orthodontics, ${ }^{3}$ Dept. of Pedodontics, Daswani Dental College Kota, Rajasthan, India \\ *Corresponding Author: \\ Email: drpashaksa@yahoo.com
}

\begin{abstract}
Introduction: It is a well known fact that the life of patient after the removal or exfoliation of all teeth becomes miserable as patient usually has common complaints of inappropriate chewing function along with some pain with the conventional dentures. The aim of this present study was to evaluate the difference in amount of bone resorption in the edentulous patients who were rehabilitated with telescopic implant denture versus those who were given screw retained prosthesis in the posterior area.

Materials and Method: The present study comprised of a total of 20 male edentulous patients from the outpatient so as to place four implants in each arch with the use of cone beam computed tomography (CBCT) and surgical technique without raising the flap. After the insertion of prosthesis, panoramic radiograph was done immediately, another after six months and one more after twelve months of using the prosthesis.

Results: The results of this present study depicted that even if the bone loss in Group I was slightly higher than Group II, but still the difference in the bone resorption in both the groups remained insignificant ( $\mathrm{p}$ value $>0.05$ ).

Discussion: As it is a well known fact that resorption of alveolar ridge occurs throughout life as it is a continuous process, so there was expected reduction in the height of alveolar ridges in the two study groups. The most probable reason for this reduction in bone height is that the transmitted forces to the alveolar bone exceed the normal level of bone tolerance.

Conclusion: This study concludes that no single treatment option is better or worse than the other and both can be used to rehabilitate the edentulous patients based upon the factors of cost of treatment, invasiveness and patient preferences.
\end{abstract}

Keywords: Telescopic crowns, Screw retained prosthesis, Bone resorption.

\section{Introduction}

It is a well known fact that the life of patient after the removal or exfoliation of all teeth becomes miserable as patient usually has common complaints of inappropriate chewing function along with some pain with the conventional dentures. ${ }^{1}$ Along with these complaints, this condition is also lowers the self esteem of the patient as well as reduction in normal functional efficiency. Nowadays another treatment modality called Osseointegrated implant supported prosthesis has come into being which has elevated the life of such patients as compared to the conventional dentures. ${ }^{2}$

The most commonly used implants are endosteal implants which are available in a variety of lengths, designs, widths and materials. These implants are very well tolerated by the patient and because of its so many advantages, it has become the first choice of the patient. $^{3}$ As this treatment modality offers good retention with wide force distribution along with good post operative healing after an easy surgery, it can be considered as a realistic choice for the replacement of teeth. ${ }^{4}$

Another treatment modality for edentulous patients is telescopic crowns which are also known as double crown. ${ }^{5}$ Such crowns are formed of primary or inner telescopic coping which is permanently attached to an abutment and an outer or secondary telescopic crown which is detachable and is connected rigidly to a detachable prosthesis. ${ }^{6}$
There are artificial teeth with acrylic resin base connected to metal framework in screw retained implant prosthesis. ${ }^{7}$ To support cantilever prosthesis, mostly 4-5 implants are placed in anterior region. In poor quality of bone, a shorter cantilever is preferred but otherwise the cantilever is supposed to be 1.5 times the antero-posterior span. ${ }^{8}$ Screw retained prosthesis is required to replace soft tissue and bone in cases of moderate to severe bone loss and this type secures the retention of prosthesis. Many trials have been done till now so as to find a solution for minimizing the bone resorption in the area of edentulous ridges and most of these trials are totally dedicated to this problem solely. ${ }^{9}$

For both the clinician and the patient, the problem of alveolar ridge resorption is very frustrating. The patients who are wearing conventional complete dentures are mostly unsatisfied by their prosthesis. With the advent of dental implants, the satisfaction level of the patients with their prosthesis increased independent of the type of prosthesis like fixed prosthesis or fixed detachable appliance or removable overdenture. ${ }^{10,11}$

The aim of this present study was to evaluate the difference in amount of bone resorption in the edentulous patients who were rehabilitated with telescopic implant denture versus those who were given screw retained prosthesis in the posterior area.

\section{Materials and Method}

The present study comprised of a total of 20 male edentulous patients from the outpatient so as to place 
four implants in each arch with the use of cone beam computed tomography (CBCT) and surgical technique without raising the flap. Eight implants (four in each arch) were placed in each one of the 20 edentulous patients which were later on prosthetically restored by either a screw retained prosthesis or a telescopic prosthesis. In this study, the difference in amount of bone loss was measured in the mandibular arch only as the bone loss in maxillary arch was observed by another team.

The patients for this study were selected from the outpatient department based upon the following inclusion and exclusion criteria:

\section{Inclusion criteria}

1. Patient should have been edentulous for at least 1 year.

2. The range of age between 50-80 years.

3. Bone thickness should be adequate as felt on palpation.

4. Appropriately covered by keratinized mucosa.

5. Maxilla-mandibular relation should be class I

\section{Exclusion criteria}

1. Presence of any pathological lesion or flabby tissue which would impede the placement of the prosthesis.

2. Patient suffering from some systemic disease like hypertension, osteoporosis, neuromuscular disorders or uncontrolled diabetes which may affect the placement and osseointegration of implants.

Bilateral canine and $2^{\text {nd }}$ premolar were the sites which were selected for placement of implants based upon identification by radiolucent channels, radiographic templates prepared previously and sufficient bone evaluation in terms of buccolingual width and bone height.

The implant which was proposed had a diameter of $3.75 \mathrm{~mm}$ and a length of $12 \mathrm{~mm}$ and $10 \mathrm{~mm}$ for canine and premolar region respectively. To check for the parallelism between the implants, four virtual implants were placed in each arch.

Before the surgery, tapered threaded, root formed implants were prepared and the patient was given a prophylactic dose of $600 \mathrm{mg}$ Ibuprofen and $2 \mathrm{gm}$ of Amoxycillin one hour prior. Dental implants were also prepared according to dimension of $3.75 \mathrm{~mm}$ diameter and a length of $12 \mathrm{~mm}$ and $10 \mathrm{~mm}$ in canine and premolar region respectively. A surgical stent was used to again locate the position of implants after 4 months of healing period using a tissue punch. Using a torque rachet, permanent transmucosal titanium abutments were placed with a torque of $40 \mathrm{Ncm}$ over the implant fixtures. Windows were cut over the implant sites in acrylic custom trays which were constructed on study casts. Open impression technique was used as final impression making after screwing the impression transfer copings to the abutment using long fixation screws.
Then using a computerized random allocation program, the patients were equally allocated in two groups. The patients in first group were rehabilitated with the use of Screw retained fixed restoration whereas the other group got Telescopic removable implant overdentures.

\section{Group I- Screw retained fixed restoration}

For checking the passivity, acrylic verification jig was formed using single screw test. Using a torque wrench, prosthesis was screwed intraorally. Lastly, using rubber pieces, the access holes were partially blocked which were later completely blocked with the help of light cured composite resin.

\section{Group II- Telescopic removable implant overdentures}

To obtain separate removable dies for analogue, the obtained casts were cut and anti-rotational plastic cap was attached to it. To get a refractory cast, the obtained cast was modified and duplicated and wax pattern of metallic framework with secondary copings were formed. The wax pattern was in the form of mesh work covering the residual ridge but was a little shorter than acrylic resin denture base.

After the insertion of prosthesis, panoramic radiograph was done immediately, another after six months and one more after twelve months of using the prosthesis. Panoramic radiographs were done with the help of Gendex Orthoralix 9200 digital machine. Prosthesis of screw retained prosthesis was fixed in the mouth while taking the radiograph whereas conventional acrylic complete dentures were there in telescopic crowns and while the patient closed the mouth in centric occlusion, radiographic exposure was made.

After obtaining the digital radiographs, the mean values of right and left side were compared and resorption of bone height was calculated using substraction radiography by comparing the base line radiographs with radiographs obtained after six months and those obtained after 12 months of prosthesis use.

\section{Results}

The present study initially consisted of twenty patients but out of the total patients, only eighteen attended the six month and twelve month follow up appointment after using the prosthesis. Two patients dropped out from the study one each from screw retained group I and telescopic overdenture group II as one of the patients had died and the other one didn't attended the follow up appointment. The reduction of alveolar bone height was recorded at each follow up appointment in both the groups. There was no statistical difference in the mean alveolar bone heights in both right and left side when compared between both the groups. That is why, the addition of mean values of decrease in bone height of both right and left sides in 
the area of posterior mandible was done at different follow up periods in each group i.e base line, after six months of prosthesis use and after twelve months of prosthesis use.

The standard deviations and mean values for the description of the data for proportional areas of posterior lower ridge height and later on the changes in height of posterior ridge were calculated at all the three follow up timings.

At baseline, first follow up and the second follow up for Group I (screw retained prosthesis), the measurements of mean values and standard deviations were $12.68 \pm 2.1 \mathrm{~mm}, 12.13 \pm 2.0 \mathrm{~mm}$ and $12.10 \pm 2.2$ $\mathrm{mm}$ respectively. At baseline, first follow up and the second follow up for Group II (telescopic overdentures), the measurements of mean values and standard deviations were $11.58 \pm 2.5 \mathrm{~mm}, 11.55 \pm 2.0$ $\mathrm{mm}$ and $11.51 \pm 1.8 \mathrm{~mm}$ respectively (Fig. 1). The results of this present study depicted that even if the bone loss in Group I was slightly higher than Group II, but still the difference in the bone resorption in both the groups remained insignificant ( $\mathrm{p}$ value $>0.05$ ).

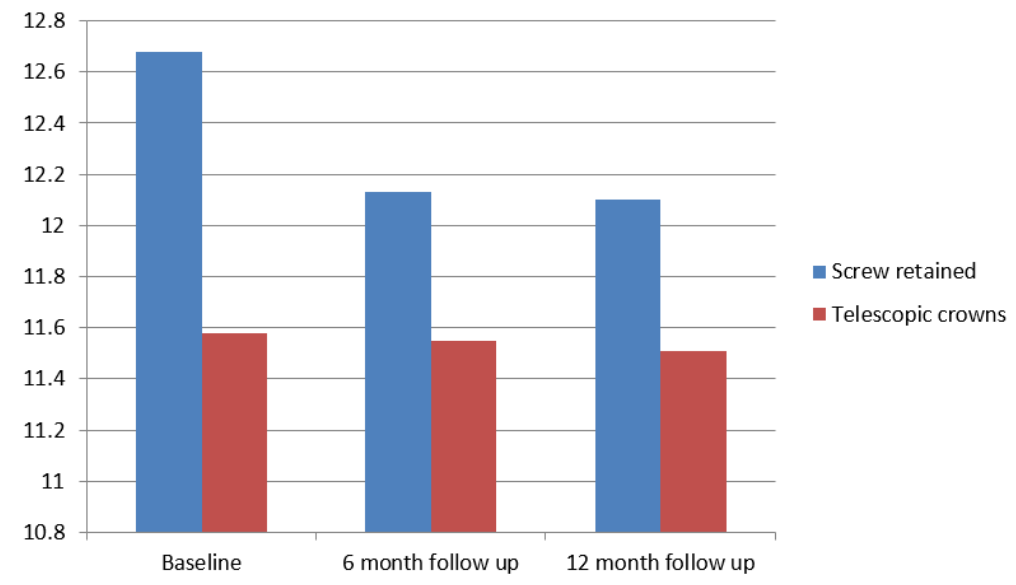

Fig. 1: Chart representing difference in bone height at different follow ups

The baseline, six months follow up and twelve months follow up bone height measurements were recorded. For Group I (screw retained prosthesis), the mean loss of bone height from baseline to $1^{\text {st }}$ follow up was $0.35 \pm 0.12$ $\mathrm{mm}^{2}$ whereas the bone loss from $1^{\text {st }}$ follow up to $2^{\text {nd }}$ follow up was $0.13 \pm 0.10 \mathrm{~mm}^{2}$ and from baseline to $2^{\text {nd }}$ follow up was $0.47 \pm 0.14 \mathrm{~mm}^{2}$. For Group II (telescopic overdentures), the mean loss of bone height from baseline to $1^{\text {st }}$ follow up was $0.23 \pm 0.07 \mathrm{~mm}^{2}$ whereas the bone loss from $1^{\text {st }}$ follow up to $2^{\text {nd }}$ follow up was $0.16 \pm 0.30 \mathrm{~mm}^{2}$ and from baseline to $2^{\text {nd }}$ follow up was $0.37 \pm 0.18 \mathrm{~mm}^{2}$ (Fig. 2). The factor of bone loss was also higher in Group I as compared to Group II but the difference was insignificant statistically ( $\mathrm{p}$ value $>0.05$ ).

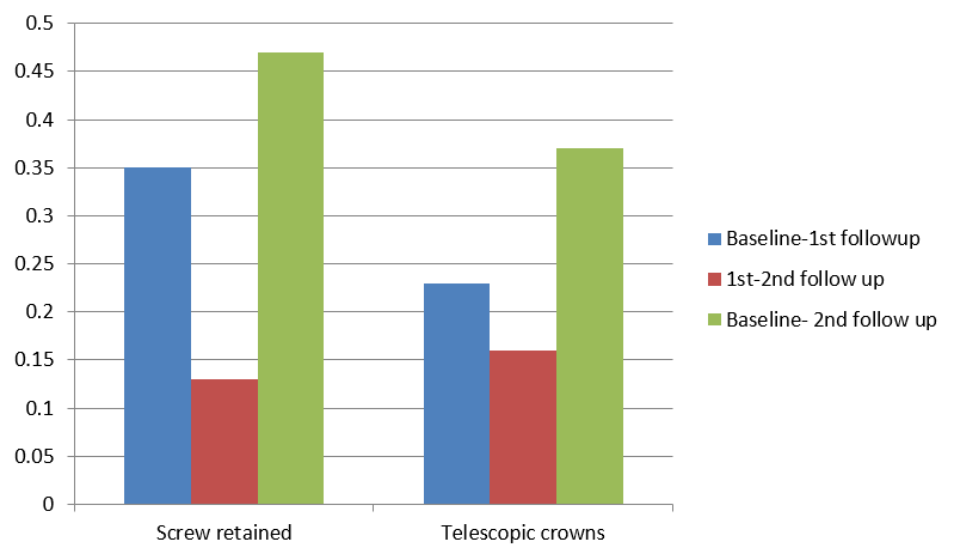

Fig. 2: Chart showing mean bone height changes at different follow ups in both groups

\section{Discussion}

As it is a well known fact that resorption of alveolar ridge occurs throughout life as it is a continuous process, so there was expected reduction in the height of alveolar ridges in the two study groups. ${ }^{12,13}$ The most probable reason for this reduction in bone height is that the transmitted forces to the alveolar bone exceed the normal level of bone tolerance. Also there can be change in the environment of supporting structures and soft tissues due to wearing 
of dentures with time and ultimately leading to bone height reduction. ${ }^{14-16}$

However in the two study groups, the reduction in alveolar ridge was not the same. Even if the difference was statistically non-significant, still there was a little higher reduction of bone height in screw retained group I as compared to telescopic crown group II which can be attributed to the reason that the patients applied greater masticatory forces in screw retained due to the feeling of natural teeth. Difficulty in maintaining the proper oral hygiene below the prosthesis fixed by screws seems to be another reason for increased bone loss. Another reason for higher bone loss in screw retained could be that there was no stimulation to alveolar ridge by food and tongue leading to greater inflammation thereby resulting in more resorption of alveolar bone.

The telescopic overdentures on the other hand gave the patient a good chance to the tissues so as to rebound and recoil and maintain his oral hygiene because these overdentures were removed during night time while sleeping. As these patients knew that the overdentures were removable, so they practiced extra care while chewing with them and did not exert too high forces which could lead to higher bone resorption.

As there were no significant differences in bone loss in between two different study groups, it is not recommended to give priority to one option over the other in rehabilitation of edentulous patients but still further studies with bigger sample size and longer follow up period are required to validate the results of this study.

\section{Conclusion}

Keeping the limitations of this present study in mind, this study concludes that no single treatment option is better or worse than the other and both can be used to rehabilitate the edentulous patients based upon the factors of cost of treatment, invasiveness and patient preferences.

\section{References}

1. Helal E, El-Zawahry M, Gouda A, Elkhadem A, Ibrahim S. Bone Height Changes of the Mandibular Edentulous Ridge in Screw Retained Versus Telescopic Restorations for Completely Edentulous Patients. Open Access Macced J Med Sci 2017;5(1):72-8.

2. Helal E, Esmat A, Gouda A, Lotfy A, El-Zawahry M. Does Implant Fixed Restoration Can Reverse The Physiological Route Of Mandibular Bone Resorption Of The Posterior Edentulous Area For The Completely Edentulous Patients?: Systematic Review. Ann Int Med Dent Res 2017;3(6):47-52.

3. Mertens C, Steveling H, Stucke K, Pretzl B, MeyerBäumer A. Fixed Implant-Retained Rehabilitation of the Edentulous Maxilla: 11-Year Results of a Prospective Study. Clin Implant Dent Relat Res 2012;14(6):816-27.

4. Yan Q, Xiao L, Su M, Mei Y, Shi B. Soft and Hard Tissue Changes Following Immediate Placement or Immediate Restoration of Single-Tooth Implants in the
Esthetic Zone: A Systematic Review and Meta-Analysis. Int J Oral Maxillofac Implants. 2016;31(6):1327-40.

5. Barone A, Toti P, Quaranta A, Derchi G, Covani U. The Clinical Outcomes of Immediate Versus Delayed

Restoration Procedures on Immediate Implants: A Comparative Cohort Study for Single-Tooth Replacement. Clin Implant Dent Relat Res 2014;17(6):1114-26.

6. Takashima M, Arai Y, Kawamura A, Uoshima K. Risk factors associated with post-loading implant loss of removable and fixed implant-supported prostheses in edentulous jaws. J Prosthodont Res 2018;62(3):365-9.

7. Ural C, Bereket C, Sener, Aktan A, Akpinar Y. Bone height measurement of maxillary and mandibular bones in panoramic radiographs of edentulous patients. J Clin Exp Dent 2011;e5-e9.

8. Mijiritsky E, Lorean A, Mazor Z, Levin L. Implant Tooth-Supported Removable Partial Denture with at Least 15-Year Long-Term Follow-Up. Clin Implant Dent Relat Res 2013;17(5):917-22.

9. Sailer I, Mühlemann S, Zwahlen M, Hämmerle C, Schneider D. Cemented and screw-retained implant reconstructions: a systematic review of the survival and complication rates. Clin Oral Implants Res 2012;23:163201.

10. Kosmin S. Advantages of Combined Cemented and Screw-Retained Implant Supported Prosthesis. A Clinical Report. J Dent Health Oral Disord Ther 2015;2(1):00037.

11. Al Amri M. Management of a Fractured Multiunit Maxillary Implant-Supported Fixed Prosthesis with Stripped Abutment Screws Using a Hybrid CementRetained and Screw-Retained Design: A 5-Year FollowUp Clinical Report. J Prosthet Dent 2015;25(4):330-4.

12. Sherif S, Susarla H, Kapos T, Munoz D, Chang B, Wright R. A Systematic Review of Screw- versus CementRetained Implant-Supported Fixed Restorations. J Prosthet Dent 2013;23(1):1-9.

13. Zurdo J, Romão C, Wennström J. Survival and complication rates of implant-supported fixed partial dentures with cantilevers: a systematic review. Clin Oral Implants Res 2009;20:59-66.

14. Eckert S. Marginal discrepancy of screw-retained and cemented metal-ceramic crowns on implant abutments. J Prosthet Dent 1999;82(6):A1.

15. Tosches N, Brägger U, Lang N. Marginal fit of cemented and screw-retained crowns incorporated on the Straumann (ITI)®Dental Implant System: an in vitrostudy. Clin Oral Implants Res 2009;20(1):79-86.

16. Alqutaibi A. Cement- and Screw-Retained ImplantSupported Restorations Showed Comparable Marginal Bone Loss and Implant Survival Rate. J Evid Based Dent Pract 2017;17(2):107-9. 